\title{
O PADRÃO 4 DE GLEASON E O VOLUME TUMORAL NO PROGNÓSTICO DO CARCINOMA DA PRÓSTATA
}

Katia R. M. Leite*, Miguel Srougi, José Roberto Kauffmann, Ruy G. Bevilacqua, Adriano J. Nesrallah, luciano J. Nesrallah, Luiz H. Camara-lopes Trabalho realizado no laboratório de Patologia Cirúrgica e Molecular, Hospital Sírio Libanês; divisão de Urologia do departamento de Cirurgia da Universidade Federal de São Paulo e departamento de Cirurgia da Universidade de São Paulo, SP.

* Correspondência: Rua Adma Jafet, 91, São Paulo, SP, Cep: 01308-050, Fone: (11) 3155-0556,

Fax: (11) 3231-2249

katiaramos@uol.com.br

\section{RESUMO}

OвJETIVos. A introdução de terapia adjuvante pós-prostatectomia radical foi recentemente proposta na literatura na tentativa de se obter melhores taxas de sobrevida em pacientes com câncer de próstata com maior risco de recidiva da doença. Alguns parâmetros anatomopatológicos têm sido considerados bons determinantes dos riscos de recorrência local ou à distância desses tumores. Recentemente o volume tumoral e a presença de padrão terciário de Gleason menos diferenciado foram apresentados como os melhores indicadores do comportamento do carcinoma da próstata. A proposta deste estudo é avaliar a importância da presença e porcentagem do padrão 4 de Gleason e do volume tumoral na evolução de pacientes portadores da adenocarcinoma bem diferenciado de próstata, tratados com prostatectomia radical.

Métodos. Setenta e sete pacientes portadores de adenocarcinoma bem diferenciado da próstata, Gleason 6 ou menos, submetidos a prostatectomia radical entre 1995 e 1997 foram estudados. Trinta e sete pacientes sofreram recidiva bioquímica (PSA $>0,4 \mathrm{ng} / \mathrm{ml})$, e 40 pacientes permaneceram livres de doença após seguimento mínimo de cinco anos. A presença e porcentagem do padrão 4 de Gleason, a porcentagem de tumor comprometendo a glândula (considerado como "volume tumoral"), a infiltração capsular e a invasão do tecido extraprostático foram submetidos a análise uni e multivariada para determinação da associação destes parâmetros com a recidiva bioquímica.

RESULTADOS. O volume tumoral foi o parâmetro mais importante para determinação da recorrência bioquímica em análises uni e multivariadas. A mediana do volume foi de $25 \%$ nos pacientes que sofreram recidiva e $11,5 \%$ naqueles que permaneceram livres de doença $(p=0,003)$. A porcentagem de padrão 4 de Gleason foi importante apenas em análise univariada. A mediana da porcentagem de Gleason 4 foi de $7,5 \%$ para os pacientes que não sofreram recidiva e de $19 \%$ naqueles que recidivaram $(p=0,046)$.

ConcLusão. O volume do adenocarcinoma de próstata é um parâmetro objetivo, de fácil avaliação e importante na previsão da recidiva bioquímica no carcinoma bem diferenciado da próstata. Por outro lado, a porcentagem do padrão menos diferenciado de Gleason também serve para prever recidiva à distância. Ambos os parâmetros devem ser incorporados em estudos futuros de terapias adjuvantes para o carcinoma da próstata.

UnITERMOS: Neoplasias prostáticas. Prognóstico. Antígeno prostático específico. Patologia. Prostatectomia. Histologia.

\section{INTRODUÇÃO}

Aproximadamente $20 \%$ dos pacientes inicialmente tratados por adenocarcinoma de próstata com prostatectomia radical, radioterapia ou crioterapia necessitam de uma segunda forma de tratamento no período de três anos após a terapia inicial'. A identificação dos casos com alta probabilidade de recidiva da doença, que podem se beneficiar de um tratamento adjuvante, é um desafio discutido atualmente para o melhor manejo do paciente com câncer de próstata. Alguns autores sustentam que a radioterapia de resgate deva ser iniciada nos primeiros sinais de recidiva naqueles pacientes tratados com cirurgia ${ }^{2}$.

Alguns parâmetros clínicos e anatomopatológicos têm sido propostos como indicadores de maior risco de recidiva. Porém, a maioria dos estudos inclui pacientes com tumores de alto grau e avançados. Não existem estudos que contemplem apenas adenocarcinomas bem diferenciados.
Partin et al. propuseram um modelo de previsão da evolução da doença, que inclui os níveis pré-operatórios do antígeno prostático espeć́fico (PSA) e, o escore de Gleason do espécime cirúrgico, quando a doença éórgão-confinada ${ }^{3}$. D' Amico et. al., ainda, incluem a invasão tumoral capsular ${ }^{4}$.

Sabe-se que o escore de Gleason é um poderoso marcador prognóstico, mas que apenas considera os dois padrões mais abundantes presentes na neoplasia. Aihara et al. descreveram a ocorrência de pelo menos três diferentes padrões de Gleason em mais de $50 \%$ dos adenocarcinomas da próstata ${ }^{5}$. Um artigo recente analisou retrospectivamente 379 pacientes submetidos a prostatectomia radical e demonstrou que a presença e a porcentagem dos padrões 4 ou 5 de Gleason foram os parâmetros mais importantes na determinação do comportamento da doença ${ }^{6}$. Pan et al. mostraram que mesmo pequenos focos dos padrões 4 ou 5 Gleason alteram a evolução desses pacientes. Neste estudo, os tumores com escore de 
Gleason 5 ou 6 que possuíam pelo menos $5 \%$ do padrão 4 de Gleason se comportaram como os adenocarcinomas escore de Gleason $7^{7}$. Stamey et al. elegantemente demonstraram que a cada incremento de $10 \%$ do padrão 4 de Gleason correspondia igual diminuição na probabilidade de sobrevida livre de doença em pacientes submetidos a prostatectomia radical ${ }^{6}$.

A importância do volume tumoral tem sido discutida na literatura desde que $\mathrm{McNeal}$ et al. associaram o volume tumoral com o potencial metastático dos adenocarcinomas da próstata. Um estudo morfométrico de 68 pacientes submetidos a prostatectomia radical mostrou a ausência de metástases linfonodais em tumores menores que $3 \mathrm{cce}$ que $100 \%$ dos tumores com comprometimento de linfonodos, $94 \%$ dos tumores com comprometimento das vesículas seminais e $100 \%$ dos tumores metastáticos para os ossos acompanhavam-se de lesões primárias maiores que $3 \mathrm{cc}^{8}$. Em análise multivariada, considerando idade, estádio patológico, penetração capsular, escore de Gleason, volume tumoral, invasão de vesículas seminais e metástases linfonodais, Norberg et al. demonstraram que o volume tumoral foi o único fator significativamente associado à progressão do câncer de próstata? .

O propósito do nosso estudo é avaliar a importância da presença e porcentagem do padrão 4 de Gleason e volume tumoral na determinação do prognóstico de pacientes tratados com prostatectomia radical por carcinoma bem diferenciado da próstata. Em outras palavras, definir se o volume tumoral e a ocorrência de padrão 4 de Gleason permitem identificar pacientes com tumores de baixo risco mas com maior chance de recorrência da doença.

\section{MÉtodos}

Este é um estudo retrospectivo, transversal. De um grupo de 375 pacientes submetidos a prostatectomia radical entre janeiro de 1995 a setembro de 1997, 235 pacientes apresentaram escore de Gleason menor ou igual a 6. Deste grupo foram escolhidos aleatoriamente 77 pacientes, 37 que sofreram recidiva bioquímica e 40 que permaneciam sem doença até o momento do estudo. Não houve nenhuma recidiva no primeiro ano pós-cirurgia e nenhum paciente recebeu terapia adjuvante antes da recidiva. Um único patologista avaliou todas as lâminas correspondentes às próstatas que foram submetidas na sua totalidade para avaliação de volume, invasão de cápsula e comprometimento extraprostático. As vesículas seminais, margens do colo vesical e uretra prostática, além dos linfonodos da fossa obturadora, foram sempre representados. O escore de Gleason foi usado para determinação da diferenciação histológica ${ }^{10}$. A porcentagem de tumor comprometendo a glândula foi avaliada segundo as descrições de Humphrey et al.", e será designada como "volume tumoral". Brevemente um gradil milimetrado foi colocado abaixo das lâminas, cujaárea ocupada pelo tumor foi previamente demarcada com caneta de tinta indelével. A porcentagem final é o resultado da média aritmética obtida após o exame de todas as lâminas. O mesmo procedimento foi utilizado para avaliação da proporção relativa do padrão 4 de Gleason. Para o estadiamento, foi utilizado o sistema TNM 2002.

Os níveis séricos de PSA foram avaliados a cada seis meses por imunoensaio (Abbott AxSYM PSA, Abbott Laboratories, Diagnostics Division, Abbott Park, IL) e a recorrência foi definida como níveis superiores a $0,4 \mathrm{ng} / \mathrm{ml}$. Para análise estatística da relação entre o volume tumoral, porcentagem de padrão 4 de Gleason e níveis de PSA, foi utilizado o valor do PSA no momento da determinação da recidiva.

Análises estatísticas foram feitas com o programa SPSS, versão 10 (SPSS, Inc. Chicago, IL). A análise univariada foi realizada por testes não paramétricos de Mann-Whitney e Kruskal-Wallis. Para análise multivariada, foram realizados testes de regressão logística. Os níveis de significância considerados para rejeição da hipótese de nulidade foram de $5 \%(p<0,05)$.

\section{Resultados}

A idade mediana dos pacientes foi de 63 anos (variável de 46 a 76 anos). Sessenta e cinco pacientes (84\%) foram estadiados como pT2. A invasão capsular foi identificada em 43 pacientes (55,8\%). Em três pacientes (4\%) foi detectada invasão do tecido extraprostáticoe foram estadiados como pT3a. Em nove (I2\%), houve invasão das vesículas seminais, sendo estadiados como $\mathrm{pT} 3 \mathrm{~b}$. Nenhum paciente apresentou metástases em linfonodos.

O escore de Gleason foi de 4 em nove pacientes (I2\%), de 5 em 21 casos (27\%) e de 6 em 43 pacientes (56\%). Em quatro casos $(5 \%)$ o escore de Gleason se alterou após a revisão das lâminas, sendo o diagnóstico final Gleason 8 . Estes casos permaneceram no estudo pois distribuíram-se igualmente entre os dois grupos que sofreram ou não recidiva bioquímica. Considerandose o próprio desenho do estudo que apenas selecionou pacientes com baixo escore de Gleason, demonstramos que não houve diferença do mesmo entre os grupos com ou sem recidiva bioquímica. A mediana do escore de Gleason foi 6 em ambos os grupos e a média foi de 5,6 naqueles com recidiva e de 5,4 naqueles sem recidiva ( $p=0,375 ; t=-0,892)$. Os resultados sobre as porcentagens do padrão 4 de Gleason e volume tumoral nos dois grupos estudados estão referidos na Tabela I.

Padrão 4 de Gleason foi encontrado em $6 \mathrm{I}$ pacientes (76,6\%). Destes, 29 (50,8\%) apresentaram recidiva. Dos I 8 casos (23,4\%) sem padrão 4 de Gleason presente, sete (38,9\%) apresentaram recorrência bioquímica.

A análise de regressão logística considerando idade, escore de Gleason, porcentagem de padrão 4 de Gleason, volume tumoral, invasão capsular e infiltração tumoral extraprostática mostrou que o único indicador de progressão foi o volume tumoral $(p=0,0 \mid 0)$. A porcentagem do padrão 4 de Gleason obteve apenas significância marginal $(p=0,058)$. Os resultados estão descritos na Tabela 2.

Quando os pacientes foram separados em três categorias de PSA no momento da recidiva $(0-0,4 \mathrm{ng} / \mathrm{ml}, 0,4-\mathrm{I}, \mathrm{I} \mathrm{ng} / \mathrm{ml}$ e $>I, \mathrm{I}$ $\mathrm{ng} / \mathrm{ml})$, a porcentagem do padrão 4 de Gleason e o volume tumoral atingiram níveis de significância estatística (Tabela 3). Enquanto o volume tumoral foi capaz de prever a recidiva em qualquer nível de PSA (Gráfico I), a porcentagem do padrão 4 de Gleason distinguiu pacientes que recidivaram com maiores níveis de PSA, que podem corresponder aos indivíduos com maior probabilidade de recidiva sistêmica (Gráfico 2). 
Tabela I - Análise univariada da porcentagem do padrão 4 de Gleason e 0 volume tumoral nos pacientes que sofreram ou não recidiva bioquímica

\begin{tabular}{|c|c|c|c|}
\hline Grupo & $\mathbf{N}$ & $\begin{array}{c}\% \text { de padrão } 4 \\
\text { mediana (variação) } \\
\end{array}$ & $\begin{array}{c}\text { \% de volume tumoral } \\
\text { mediana (variação) }\end{array}$ \\
\hline Comrecidiva & 37 & $19,0(0-100)^{*}$ & $25,0(5-70)^{* * *}$ \\
\hline Semrecidiva & 40 & $7,5(0-100)^{*}$ & $11,5(3-45)^{* * *}$ \\
\hline
\end{tabular}

\section{Tabela 2 - Regressão logística considerando idade, escore de Gleason, porcentagem do padrão 4 de Gleason, volume tumoral, infiltração da cápsula e invasão do tecido extraprostático nos grupos de pacientes que sofreram ou não recidiva bioquímica}

\begin{tabular}{lc}
\hline Variáveis & Significância(2-bicauldal) \\
\cline { 2 - 2 } Idade & 0,137 \\
Escorede Gleason & 0,324 \\
Gleason4(\%) & 0,058 \\
Infiltraçãodacápsula & 0,392 \\
Invasãoextraprostática & 0,129 \\
Volume(\%) & $0,010^{*}$ \\
\hline
\end{tabular}

*ignificante

Tabela 3 - Análise univariada, teste de Kruskal-Wallis para grupos com diferentes níveis de PSA no momento da recidiva bioquímica

\begin{tabular}{|c|c|c|c|}
\hline $\begin{array}{l}\text { Níveis séricos de } \\
\text { PSA }(\mathrm{ng} / \mathrm{ml})\end{array}$ & $\mathbf{N}$ & $\begin{array}{l}\text { \% de padrão } 4 \\
\text { mediana } \\
\text { (variação) }\end{array}$ & $\begin{array}{l}\text { \% volume tumoral } \\
\text { mediana } \\
\text { (variação) }\end{array}$ \\
\hline $0-0,4$ & 40 & $7,5(0-100)^{*}$ & II,5(3-45)*** \\
\hline $0,4-1,1$ & 17 & $12,0(0-100) *$ & $25,0(5-65) * *$ \\
\hline$>1,1$ & 20 & $31,5(0-100)^{*}$ & $25,0(5-70) * * *$ \\
\hline
\end{tabular}

${ }^{*} \mathrm{p}=0,001{ }^{*}{ }^{*} \mathrm{p}=0,013$

Gráfico I - Volume tumoral entre os grupos com diferentes níveis de PSA no momento da recidiva bioquímica. Os dados indicam diferença entre os grupos sem recidiva bioquímica e aqueles com recidiva, em qualquer nível de $\mathrm{PSA}(\mathrm{p}=0,020)$

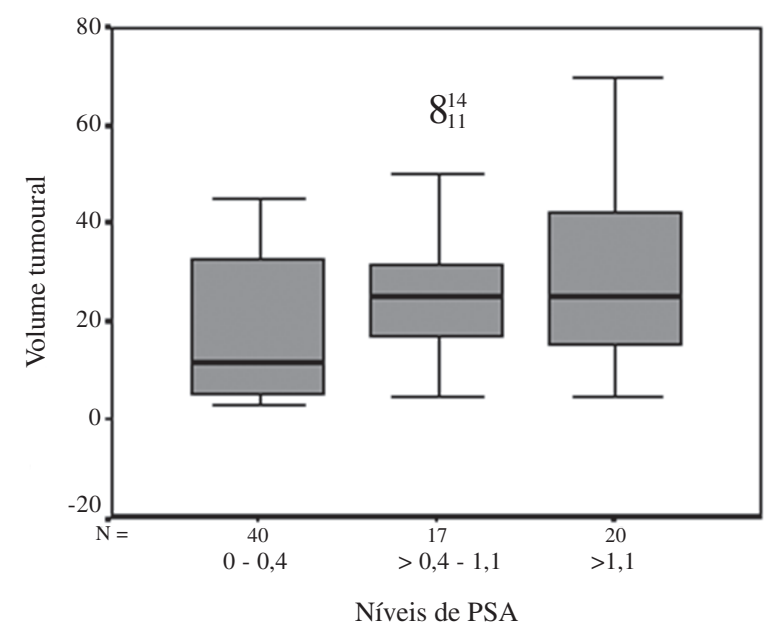

Gráfico 2 - A porcentagem de padrão 4 de Gleason entre os três grupos de PSA. Os dados indicam diferença em relação ao grupo que apresentou maiores níveis de PSA no momento da recidiva $(p=0,002)$

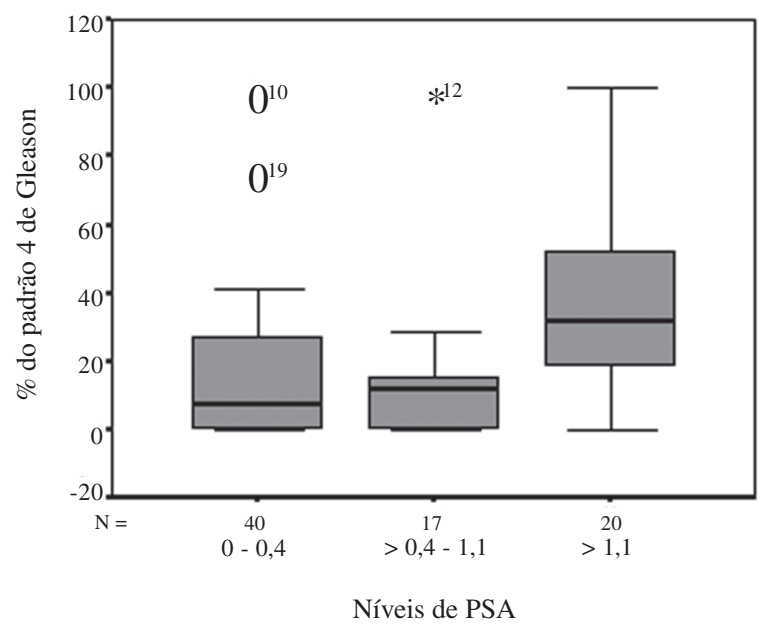

\section{Discussão}

Considerando o PSA superior a $0,4 \mathrm{ng} / \mathrm{ml}$ como ponto de corte para definição de progressão bioquímica, como recentemente proposto por Amling etal. ${ }^{12}$, nosso estudo demonstrou que o volume tumoral foi o fator prognóstico mais importante nos adenocarcinomas bem diferenciados da próstata, tanto em análise uni quanto multivariada. A mediana do volume para pacientes que sofreram recidiva foi de $25 \%$ e de apenas II,5\% para aqueles que permaneceram sem a doença.

Já se conhece bem a correlação entre volume tumoral e estádio patológico do adenocarcinoma da próstata ${ }^{8}$. 0 volume tumoral constitui uma informação objetiva, independemente da experiência do patologista e pode ser analisado com facilidade, assim como descrito por Humphrey and Vollmer". Recentemente, Carvalhal et al. avaliaram visualmente o volume tumoral em espécimes de prostatectomia radical de 595 homens e demonstraram que para cada incremento de $5 \%$ no volume tumoral existe um incremento de II\% na probabilidade de recidiva do tumor ${ }^{13}$. Contrariamente ao nosso resultado, Herman et al., em análise multivariada de 823 pacientes, não observaram influência significativa do volume tumoral em relação à recidiva tumoral ${ }^{14}$. Demonstraram que o status da margem cirúrgica, extensão extraprostática, metástases para os linfonodos, comprometimento de vesículas seminais e os níveis pré-operatórios de PSA foram mais importantes na determinação do prognóstico. A diferença em relação ao nosso trabalho está na seleção dos pacientes. Nosso estudo selecionou apenas tumores bem diferenciados, Gleason 6 ou menos, enquanto o deles incluía apenas adenocarcinomas Gleason 7, 67\% deles não eram órgãoconfinados e 7,7\% dos pacientes tinham neoplasia metastática em linfonodos, indicando que, na presença de componente 4 de Gleason, ou de outros fatores desfavoráveis, o volume tumoral perde a sua relevância. 
Nossos resultados mostraram a importância prognóstica do volume tumoral principalmente nos carcinomas que não possuíam o padrão 4 de Gleason na sua histologia. Entre 18 pacientes cujo adenocarcinoma não possuía nenhuma área 4 de Gleason, sete $(38,9 \%)$ sofreram recorrência bioquímica e o volume mediano de tumor neste grupo foi significativamente maior do que naqueles pacientes que não sofreram recidiva.

Mesmo em biópsias de próstata, a avaliação do volume tumoral parece ser importante na determinação do prognóstico. Um artigo recente mostrou que a porcentagem de tumor presente em biópsias feitas em 190 pacientes foi o fator mais significante para prever a recidiva bioquímica ${ }^{15}$.

Na nossa série, a porcentagem do padrão 4 de Gleason foi importante determinante prognóstico apenas em análise univariada. A porcentagem mediana do padrão 4 de Gleason foi de $7,5 \%$ em pacientes livres de recidiva e de $19 \%$ nos pacientes que recidivaram. Nossos resultados são diferentes daqueles publicados por Stamey et al. ${ }^{6}$, que descreveram que a porcentagem dos padrões 4 e 5 de Gleason é o fator mais importante na previsão de falha bioquímica. Pan et al. mostraram que mesmo a presença de pequeno volume de componente 4 ou 5 de Gleason mudava o comportamento do tumor ${ }^{7}$. Na sua casuística, tumores escore de Gleason 6, quando tinham pelo menos $5 \%$ de padrão 4, comportaram-se como adenocarcinomas Gleason 7. O mesmo ocorreu em tumores escore 7, em que a presença de pelo menos $5 \%$ de padrão 5 alterou o comportamento do tumor, que progrediu como um adenocarcinoma pouco diferenciado, escore de Gleason 8 a 10. Mais recentemente, três estudos demonstraram que a porcentagem do padrão 4 de Gleason nos carcinomas Gleason 7 representa parâmetro importante na determinação da progressão da doença ${ }^{14,16,17}$.

A literatura tem demonstrado maiores taxas de sobrevida livre de doença e menos efeitos colaterais em pacientes tratados com radioterapia adjuvante precocemente após a prostatectomia radical, quando

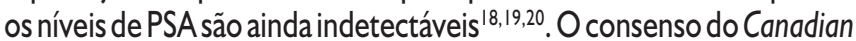
GURadiation Oncologist Group propôs a separação de pacientes em três categorias pre-tratamento: baixo risco, risco intermediário e alto risco de progressão da doença, considerando-se o escore de Gleason, os níveis de PSA e o estádio tumoral| ${ }^{21}$. D'Amico et al. consideraram previamente o estádio do tumor, o escore de Gleason, a presença de margens positivas, após a prostatectomia radical, como bons indicadores de risco de progressão de tumor ${ }^{22}$. Não incluíram no estudo a presença e porcentagem do padrão menos diferenciado de Gleason ou volume tumoral.

O momento da introdução da terapia antiandrogênica é outra questão em debate. A elaboração de ensaios clínicos tem sido encorajada por alguns autores para definir qual seria o momento mais apropriado para introdução do tratamento, se precoce ou após o início da elevação do PSA ${ }^{23}$. Alguns têm descrito melhores resultados quando a terapia é introduzida precocemente nos pacientes com tumores considerados agressivos, com alto grau de Gleason e doença metastática em linfonodo ${ }^{24,25}$. Nenhum estudo foi publicado com pacientes portadores de adenocarcinoma localizado de baixo grau histológico.

\section{Conclusão}

O nosso resultado mais interessante foi a avaliação dos grupos considerando os diferentes níveis de PSA no momento da recidiva da doença. De maneira geral, os níveis de PSA se correlacionam com a extensão da doença no momento da recorrência: níveis mais baixos associam-se a recidiva local e níveis mais altos a recidiva sistêmica ${ }^{26}$. Assim, separando os pacientes em três grupos, respectivamente com PSA $<0,4 \mathrm{ng} / \mathrm{ml}, 0,4-\mathrm{I}, \mathrm{I} \mathrm{ng} / \mathrm{ml}$ e $>\mathrm{I}, \mathrm{I} \mathrm{ng} / \mathrm{ml}$, observamos que 0 volume tumoral e a porcentagem do padrão 4 de Gleason mostraram associação com progressão da doença. Novamente, o volume tumoral distinguiu pacientes sem recorrência daqueles com recidiva, independentemente do nível de PSA. Por outro lado, a porcentagem do padrão 4 de Gleason associou-se a doença recidivada com maiores níveis de PSA, sugerindo que a porcentagem do padrão 4 de Gleason seja um indicador de recidiva sistêmica. Esta informação pode ser muito útil para o manejo dos pacientes submetidos a prostatectomia radical. Os casos com maior risco de recidiva local (grande volume tumoral e ausência ou baixa porcentagem do padrão 4 de Gleason) deveriam ser tratados com radioterapia adjuvante, e pacientes com maior risco de recidiva sistêmica (alta porcentagem de padrão 4 de Gleason) seriam melhor tratados com deprivação androgênica.

Nossos dados indicam que o desenho de ensaios clínicos para definir a indicação de radioterapia ou hormonioterapia adjuvantes deve incorporar a porcentagem do padrão 4 de Gleason e o volume tumoral, de modo a identificar os pacientes com maior risco de recidiva local ou à distância.

\section{Conflito de interesse: não há}

\section{SUMMARY}

WELL DIFFERENTIATED LOCALIZED PROSTATE CARCINOMA: Prognostic relevance of tertiary Gleason pattern 4 and TUMOR VOLUME

INTRODUCTION. Early introduction of adjuvant radiation or hormone therapy after radical prostatectomy has been advocated in recent literature aiming to improve survival rates in high risk prostate cancer. Traditional prognostic parameters such as serum PSA, Gleason score and clinical stage have been used to identify these cases however accuracy is far from ideal. Recently, tumor volume and presence and percentage of a tertiary less differentiated Gleason pattern have been considered better indicators of prostate cancer behavior. The purpose of this study was to evaluate the importance of presence and percentage of a Gleason pattern 4 and of tumor volume in the outcome of patients treated for well-differentiated prostate adenocarcinoma.

METHODS. Seventy seven patients submitted to radical prostatectomy for well-differentiated prostate carcinoma, Gleason score 6 or less, and followed up for at least 5 years were selected. Thirty seven experienced biochemical recurrence while 40 did not. The percentage of Gleason pattern 4, the percentage of the gland involvement (considered as "tumor volume"), capsular invasion and extraprostatic extension were submitted to univariate and multivariate analyses for the evaluation of possible associations with biochemical recurrence. 
RESULTS. Tumor volume was the most important parameter to predict biochemical recurrence by univariate and multivariate analysis. The median of tumor volume was $25.0 \%$ for patients who suffered recurrence, and $11.5 \%$ for those with no recurrence $(p=0.003)$. The percentage of Gleason pattern 4 was predictive of recurrence in univariate analysis only. The median percentage of Gleason pattern 4 was $7.5 \%$ for patients without recurrence and $19.0 \%$ for those who presented recurrence $(p=0.046)$.

CONCLUsion. Tumor volume is very objective, easy to evaluate and the most important parameter to predict biochemical recurrence in well-differentiated adenocarcinoma of the prostate. On the other hand, presence and percentage of a tertiary less differentiated Gleason pattern correlated with systemic relapse. Both parameters should be included in future studies to evaluate the role of adjuvant therapy in high risk prostate cancer. [Rev Assoc Med Bras 2005; 5 I (5): 329-33]

KeY wORDS: Prostatic neoplasm. Prognosis. Prostate-specific antigen. Tumor volume. Radical prostatectomy. Gleason grade.

\section{REFERÊNCIAS}

I. Grossfeld GD, Stier DM, Flanders SC, Henning JM, Schonfeld W, Warolin $\mathrm{K}$, et al. Use of second treatment following definitive local therapy for prostate cancer: data from the CAPSURE database. J Urol 1998; 160:1398-404.

2. Schild SE, BuskirkSJ, Wong WW, Halyard MY, Swanson SK, Novicki DE, et al.. The use of radiation for patients with isolated elevation of serum prostate specific antigen following radical prostatectomy. J Urol 1996; I 56: I 725-29.

3. Partin AW, Piantadosi S, Sanda MG, Epstein JI, Marshall FF, Mohler JL, et al. Selection of men at high risk for disease recurrence for experimental adjuvant therapy following radical prostatectomy. Urology 1 995;45:83I-8.

4. D 'Amico AV, Whittington R, Schultz D, Malkowicz SB, TomaszewskiJE, Wein A. Outcome based staging for clinically localised adenocarcinoma of the prostate. JUrol 1997; 1 58: 1422-6.

5. Aihara M, Wheeler TM, Ohori M, Scardino PT. Heterogeneity of prostate cancer in radical prostatectomy specimens. Urology 1994;43:60-6.

6. Stamey TA, McNeal JE, Yemoto CM, Sigal BM, Johnstone IM. Biological determinants of cancer progression in men with prostate cancer. JAMA | 999;28|: | 395-400.

7. Pan CC, Potter SR, Patin AW, Epstein JI. The prognostic significance of tertiary Gleason patterns of higher grade in radical prostatectomy specimens: a proposal to modify the Gleason grade system. Am J Surg Pathol 2000;24:563-9.

8. McNealJE, Bostwick DG, Kindrachuk RA, Redwine E, Freins FS, Stamey TA. Pattern of progression in prostate cancer. Lancet 1986; I :60-3.

9. Norberg M, Holber GL, Wheeler T, Magwsson A. Five year follow up after radical prostatectomy for localised prostate cancer: a study of the impact of different tumour variables on progression. Scand J Urol Nephrol 1994;28:391-9.

10. Gleason DF. Histologic grading of prostate cancer: a perspective. Hum Pathol 1992;23:273-9.

II. Humphrey PA, Vollmer RT. Intraglandular tumour extent and prognosis in prostatic carcinoma: Application of a grid method to prostatectomy specimens. Hum Pathol 1990;21:799-804.
12. Amling CL, Bergstralh EJ, Blute ML, Slezak JM, Zincke H. Defining prostate specific antigen progression after radical prostatectomy: what is the most appropriate cut point? J Urol 200 I; I65: I |46-5 I.

13. Carvalhal GF, Humphrey PA, Thorson P, Yan Y, Ramos CG, Catalona WJ. Visual estimate of the percentage of carcinoma is an independent predictor of prostate carcinoma recurrence after radical prostatectomy. Cancer 2000; 89:I308-I4.

14. Herman SM, Kattan MW, Ohari M, Scardino PT, Wheeler TM. Primary Gleason pattern as a predictor of disease progression in Gleason score 7 prostate cancer. Am J Surg Pathol 200 I;25:657-60.

I5. Freedland SJ, Csathy GS, Dorey F, Aronson WJ. Percent prostate needle biopsy tissue with cancer is more predictive of biochemical failure or adverse patholgy after radical prostatectomy than prostate specific antigen or Gleason score. J Urol 2002; 167:5 16-20.

16. Sakr WA, Tefilli MV, Grignon DJ, Banerjee M, Dey J, Gheiler EL, et al. Gleason score 7 prostate cancer: A heterogeneous entity? Correlation with pathologic parameters and disease-free survival. Urology 2000;56:730-34.

17. Han M, Partin AW, Pound CR, Epstein JI, Walsh PC. Long-term biochemical disease-free and cancer-specific survival following anatomic radical retropubic prostatectomy. The I5-year Johns Hopkins experience. Urol Clin North Am 200 I; 28:555-65.

18. Anscher MS. Adjuvant radiotherapy following radical prostatectomy is more effective and less toxic than salvage radiotherapy for rising prostate specific antigen. Int J Cancer 200I;96:9I-3.

19. Catton C, Gospodarowicz M, Ward P, Panzarella T, Catton P, McLean M, et al. Adjuvant and salvage radiation therapy after radical prostatectomy for adenocarcinoma of the prostate. Radiother Oncol 200 I;59:5 I-60.

20. Parker $C$, Warde $P$, Catton $C$. Salvage radiotherapy for PSA failure after radical prostatectomy. Radiother Oncol 200 I; 61:107-16.

2I. Lukka H, Warde P, Pickles T, Morton G, Brundage M, Souhami L. Controversies in prostate cancer radiotherapy: consensus development. Can J Urol 2001;8:1314-22.

22. D 'Amico AV, Whittington R, Malkowicz SB, Fondurulia J, Chen $M H$, TomaszewskiJE, et al. The combination of preoperative prostate specific antigen and postoperative pathological findings to predict prostate specific antigen outcome in clinically localised prostate cancer. J Urol 1998;160:2096-101.

23. Walsh PC, DeWeese TL, Eisenberger MA. A structured debate: immediate versus deferred androgen suppression in prostate cancer: evidence for deferred treatment. JUrol 200 I; I66:508-I5.

24. Messing EM, Manola J, Sarosdy M, Wilding G, Crawford ED, Trump D. Immediate hormonal therapy compared with observation after radical prostatectomy and pelvic lymphadenectomy in men with node-positive prostate cancer. N Engl J Med I 999; 34 I: I 78I-8.

25. Lau WK, Bergstralh EJ, Blute ML, Slezak JM, Zincke H. Radical prostatectomy for pathological Gleason 8 or greater prostate cancer: Influence of concomitant pathological variables. J Urol 2002; 1 67: I 17-22.

26. Pound CR, Partin AW, Eisenberger MA, Chan DW, Pearson JD, Walsh PC. Natural history of progression after PSA elevation following radical prostatectomy. JAMA 1999;281:1591-7.

Artigo recebido: 28/06/04 Aceito para publicação: 03/02/05 